\title{
Gait asymmetry assessment for older adults by measuring circular gait speed
}

\section{$\operatorname{AUTHOR}(\mathrm{S})$ :}

Ichihashi, Noriaki; Ikezoe, Tome; Sato, Syunsuke; Ibuki, Satoko

\section{CITATION:}

Ichihashi, Noriaki ...[et al]. Gait asymmetry assessment for older adults by measuring circular gait speed. Geriatrics and Gerontology International 2019, 19(8): 736-739

\section{ISSUE DATE:}

2019-08

URL:

http://hdl.handle.net/2433/243818

\section{RIGHT:}

This is the peer reviewed version of the following article: [Noriaki Ichihashi, Tome Ikezoe, Syunsuke Sato, Satoko Ibuki. Gait asymmetry assessment for older adults by measuring circular gait speed. 'Geriatrics and Gerontology International' 19(8), 736-739], which has been published in final form at https://doi.org/10.1111/ggi.13691. This article may be used for non-commercial purposes in accordance with Wiley Terms and Conditions for Use of Self-Archived Versions.; The fulltext file will be made open to the public on 08 August 2020 in accordance with publisher's 'Terms and Conditions for Self-Archiving'; この論文は出版社版でありません。引用の際には出版社版をご確認じ利用ください。; This is not the published version. Please cite only the published version. 
Gait asymmetry assessment for older adults by measuring circular gait speed

Noriaki Ichihashi, PT, $\mathrm{PhD}^{1 *}$

Tome Ikezoe, PT, $\mathrm{PhD}^{1}$

Syunsuke Sato $\mathrm{PT}^{1}$

Satoko Ibuki, PT ${ }^{1}$

\section{The addresses of the institutions at which the work was carried:}

Human Health Sciences, Graduate School of Medicine, Kyoto University, Kyoto, Japan 53 Kawahara-cho, Shogoin, Sakyo-ku, Kyoto 606-8507, Japan.

\section{Corresponding author:}

Noriaki Ichihashi,

Postal address: Human Health Sciences, Graduate School of Medicine, 53 Kawahara-cho, Shogoin, Sakyo-ku, Kyoto 606-8507, Japan.

Telephone: +81-75-751-3951; Fax: +81-75-751-3951

E-mail: ichihashi.noriaki.5z@kyoto-u.ac.jp

\section{Author contribution}

Noriaki Ichihashi: study concept and design, data interpretation.

Tome Ikezoe: data acquisition and analyses

Syunsuke Sato: data acquisition

Satoko Ibuki: writing of the manuscript

\section{Short running title}

Circular gait speed measurement 


\section{Abstract}

Aim: The aim of the study was to evaluate the effectiveness of the circular gait test as a method for detecting side-to-side straight-line gait asymmetry in older adults.

Methods: Twenty-five healthy older adults and 20 healthy young adults participated in this study. Walking speeds and right- and left-leg step lengths and stance durations were measured during straight-line walking using a triaxial accelerometer (G-walk from Bertec Japan Co., Ltd.). Step length and stance duration laterality were calculated using the side-to-side difference (|left - right|) and asymmetry index (|left - right|/mean right and left values $\times 100$ ). For circular gait, time required to walk twice around a 1-m diameter circle for right and left rotations was measured, and the laterality was calculated using the same formula as that described for the straight-line gait.

Results:The results indicated no differences in straight-line step length or stance duration laterality between groups. However, circular gait asymmetry was significantly higher for older subjects. Significant correlation was confirmed between circular gait time and straight-line step length for older adults $(r=0.404)$.

Conclusions: The study suggested that the circular gait test had better potential to detect age-related changes in gait laterality than straight-line gait step length or stance duration, and it may work as a simple assessment method for detecting laterality in 
straight-line walking.

Keywords: Circular gait, cost-effective, gait assessment, older adults, side-to-side asymmetry, 


\section{Introduction}

Gait asymmetry such as side-to-side difference in step length and step time contributes to risk of falls in older adults ${ }^{1-3}$. Side-to-side asymmetry leads to disruption of the medial-lateral balance. In fact, step-time asymmetry in older adults is correlated with decreased postural stability ${ }^{3}$. However, equipment such as triaxial accelerometer and video analysis are required for measurements of gait asymmetry; conducting these measurements in a busy clinical setting or in the field, is therefore difficult.

In post-stroke patients, asymmetry during straight-line gait is correlated with the turning performance while walking along a circular treadmill ${ }^{4}$. High total body stability is required for turning performance because stance duration is longer for the inner limb than for the outer limb, and higher propulsion is required for the outer limb to generate longer step lengths ${ }^{5}$.Turning performance requires coordinated movement of the lower limbs and greater control of the medial-lateral balance than during straightline gait, and is therefore a more challenging task even for healthy older adults ${ }^{6,7}$.

Based on these findings, we hypothesized that the difference in turning performance, which can be evaluated as the time taken to walk around a circular path (circular gait) in right- and left-handed directions, would be an indicator of side-to-side differences in normal straight-line gait in older adults. Circular gait is a continuation of 
turning performance, and the inner limb needs to have a longer stance phase and shorter step length than the outer limb. Therefore, it is assumed that if a side-to-side difference exists during straight-line gait in a subject, the discrepancy would be evident by measuring the time difference in circular gait while turning one way and the other way around. We hypothesized that, if the step length was shorter or the stance phase was longer in the right limb than in the left limb during straight-line gait, the time taken for the left-handed direction would be longer during circular gait. A circular gait is a more challenging for older adults compared with straight-line gait. Furthermore, a gait asymmetry could be emphasized more in the circular gait, and therefore could be effective for detecting age related changes in gait laterality. In addition, to evaluate gait asymmetry during straight-line gait, expensive equipment, such as a 3D motion analysis system and triaxial accelerometer, and a long walkway to record several steps are required. At least two examiners are needed, one to operate the equipment and another to assure the safety of the subjects, and a certain amount of time is needed to carry out the experiment. On the other hand, a circular gait test, which can be performed by one examiner using a stopwatch and a circular path, is an easy and quick experiment, which could be considered as a simple and cost-effective method to detect gait asymmetry.

The purpose of this study was to reveal the correlation between the lateralities 
during straight-line gait and in the right- and left-handed rotations around a circular

path. Furthermore, the age-related changes in gait laterality during a circular gait test is investigated for the purpose of developing a cost-effective method to detect gait asymmetry in older adults.

\section{Methods}

\section{Participants}

The subjects comprised 20 young healthy adults (age $21.5 \pm 0.9$ years, 16 women and four men) and 25 older adults (age $84.8 \pm 7.4$ years, 19 women and six men) living in nursing homes in Kyoto, Japan. Subjects were able to ambulate independently and did not present with any unstable physical condition. Exclusion criteria included: 1) presence of dementia or severe higher brain dysfunction, 2) difficulty in comprehending the study purpose or the instructions, 3) recent surgical history that impaired mobility, and 4) any severe musculoskeletal impairment (such as severe pain and rheumatoid arthritis), or neurological disorders (such as hemiparetic disease, Parkinson's disease, and paresis of the lower limbs) that limited normal walking ability. Subjects were given precise information about the content of the study. Informed consent was obtained from all subjects. This study was approved by the ethics 
committee of Kyoto University Graduate School of Medicine (E1141).

\section{Evaluation of asymmetry during walking in a straight line}

Straight-line walking asymmetry was measured using a wireless, inertial sensor that encompasses a tri-axial accelerometer, a magnetic sensor, and a tri-axial gyroscope (G-walk, Bertec Japan Co., Japan). Walking speed, step length of the left and right limbs, and the stance duration of each limb during $10 \mathrm{~m}$ of straight-line gait under comfortable walking conditions were evaluated. The stance duration of each limb was calculated by detecting the contact and lift-off of the left and right limb from the anterior-posterior acceleration signal. For instance, to calculate the stance duration of the right limb, the time of right foot contact was subtracted from the time of lift off. Step length was predicted from cadence and time of gait cycle and was corrected by the lower extremity length. The average step length and stance duration of the left and right limbs were calculated from all the steps and stances during the $10 \mathrm{~m}$. Based on the average values of the left and right limb, the longer and shorter values were determined respectively.

The straight-line walking asymmetry in the step length and stance duration were determined by the side-to-side difference and asymmetry index. The side-to-side 
difference and asymmetry index were calculated using the following equations,

Side-to-side difference $=\mid$ Left- Right $\mid$

and

Asymmetry index $=\mid$ Left - Right $\mid /$ mean value for Left and Right $\times 100$, respectively. Here, Left and Right are the values of the average step length or stance duration of the left and right limbs, respectively. In the above equation, values of 0.0 represent a perfect right-left symmetry and higher values reflect greater degrees of rightleft asymmetry.

\section{Circular gait measurement}

Using a stopwatch, circular gait was evaluated as the time taken to walk two rounds around a 1-m-diameter circle. Measurements were taken at a comfortable walking speed, and were obtained twice, each for walking in right and left rotation in a random order. A one meter diameter circle line was drawn on the floor and a starting line was marked on the circle. The examiner used the command "go" and the subjects walked around the circle line for two and a half rounds at a comfortable speed. The last half round was considered as a deceleration phase, and the time taken from the telling them to "go" until the subjects walked the second round was recorded using a 
stopwatch. The examiner walked very close to the subjects and took care that no adverse events occurred, such as a participant falling during the measurements.

The average times of the two trials for the right and left rotations were

calculated. The circular gait asymmetry was also determined by the side-to-side

difference and asymmetry index. The side-to-side difference and asymmetry index were

calculated using the following equations:

$\underline{\text { Side-to-side difference }=\mid \text { left rotation } \text { - right rotation } \mid}$

and

$\underline{\text { Asymmetry index }=\mid \text { left rotation } \text { - right rotation } \mid / \text { mean value for left and right }}$

$\underline{\text { rotation } \times 100 \text {, }}$

respectively.

\section{Statistical analysis}

The Mann-Whitney U test was used to determine the differences in gait

variables between the young and older adults. Spearman's correlation coefficient was

used for both groups to determine the relationship between side-to-side differences in

the straight-line gait measurements and circular gait time, and between asymmetry

indexes in straight-line gait and circular gait. Furthermore, Spearman's correlation 
coefficient was used to determine the relationship between the straight-line gait speed and the asymmetry indexes of straight-line gait and circular gait. P values $<0.05$ were considered significant.

\section{Results}

\section{Comparison of asymmetry in the young and older adults}

The values determined for straight-line gait and circular gait in the groups of young and older adults are compared in Table 1. For straight-line gait, no significances were found between the young and older adults for either asymmetry index in step length or in stance duration. In circular gait, the asymmetry index of the older adults $(9.06 \pm 7.05 \%)$ was significantly higher than that of the young adults group (3.58 \pm

\subsection{4\%) (Table 1).}

\section{Relationship between asymmetry in straight-line gait and circular gait}

In the young adults group, no significant relation to the asymmetry in circular gait was found with either step length or stance duration (Table 2). However, in the case of older adults, a significant correlation was found between the difference in step length and the difference in circular gait time $\quad(r=0.404)$ (Table 2). In other words, subjects who walked slower in left-handed rotation exhibited a longer step length in the left leg 
than in the right leg during straight-line gait.

\section{Relationship between straight-line gait speed and asymmetry index}

There existed no significant relationship between the straight-line gait speed and the asymmetry indexes of straight-line gait and circular gait in both young and older adults.

\section{Discussion}

Gait deficit has been well established as a risk factor for falls in older adults ${ }^{8}$.

Particularly, low gait speed ${ }^{9-11}$ and gait asymmetry ${ }^{1-3}$ have been known to be related to a high risk of falling. In this study, with an aim to develop a new simple method to detect side-to-side gait asymmetry, we evaluated the efficiency of the circular gait test. This test was based on the hypothesis that the difference in gait speed around a circular path, in right- and left-handed rotations, constitutes an indicator of side-to-side differences in normal straight-line gait.

The current study revealed no difference in the asymmetry index for step length or stance duration between the young and older adults during straight-line gait. Though there was no significant difference in straight-line gait asymmetry with age, previous studies have indicated that gait asymmetry in older adults is correlated with fall risk ${ }^{1-3}$. Therefore, we consider that screening of gait asymmetry is important in older adults. 
On the other hand, the asymmetry index of the groups differed significantly during circular gait. This indicates that, older adults exhibited greater side-to-side differences in stance duration and step length during circular gait, leading to difference in the gait time. When walking on a curved path, it is necessary to prolong the stance phase of the inner limb and increase the step length of the outer limb. It was demonstrated that such adaptation to curved paths may be difficult especially for older adults with mobility problems ${ }^{12}$. Therefore, we assumed that circular gait elicits a greater increase in asymmetry among older adults. According to our hypothesis, the gait asymmetry of older adults may be emphasized by performing a circular gait test, which suggests that the circular gait test may be more effective for detecting agerelated changes in the gait laterality, rather than the assessment of straight-line gait. The results also indicated that, in the case of the older adults, there was a significant relation between difference in step length during straight-line gait and gait time difference during circular gait. However, in the case of young adults, there was no significant relation between each index in straight-line gait and difference in circular gait time. In older adults, the side-to-side difference in step length during straight-line gait was positively correlated with the difference in circular gait time. This suggested that older adults, whose step length of the right limb is shorter than that of the left limb, 
walk more slowly in left-handed rotation than in right-handed rotation. Interestingly, our study indicated that the straight-line gait speed had no relationship with any of the asymmetry indexes during straight-line gait. The finding suggested that asymmetry during straight-line gait reflected different aspects of gait parameters from gait speed. Therefore, the difference between the left and right circular-gait time can be used as a test to determine the gait asymmetry in straight-line gait, especially the asymmetry in step length. The circular gait test does not require expensive equipment, an equipment operator, or a long walkway. It can be examined by one examiner using a stopwatch and an area in which a one meter diameter circle can be drawn. Our findings suggest that the circular gait test is a suitable cost-effective method for detecting gait asymmetry in older adults.

A limitation of this study is that the correlation coefficient was relatively low $(\mathrm{r}=0.404)$, although the asymmetry during straight-line gait is significantly correlated with the time difference in circular gait. This may imply that it needs further improvements for increased validity to detect asymmetry during straight-line gait. This low correlation results possibly from the fact that subjects with physical dysfunctions were excluded, and hence the side-to-side difference was small. Indeed, it has been 
reported that subjects with impairments such as hemiparesis, unilateral musculoskeletal injury, exhibit gait asymmetry ${ }^{13,14}$. Therefore, a greater correlation between the asymmetry in straight-line gait and circular gait is expected in subjects with these impairments. A previous study showed that 4 weeks of circular gait training reduced the side-to-side asymmetry in straight-line gait in hemiparetic subjects ${ }^{4}$. This may also apply to older adults with gait asymmetry stemming from diseases other than hemiplegia. Other limitations of this study are that we could not examine the relationship between the gait asymmetry and fall risk. As walking is the most frequently cited cause of falls ${ }^{15}$, the assessment of the gait deficit may be useful for finding methods more capable of predicting fall risk. In addition, significant differences between fallers and non-fallers can be observed, especially in more challenging conditions during postural control ${ }^{16}$. Therefore, asymmetries during circular gait could potentially be used to discriminate between fallers and non-fallers in the older adult group. Further study is required to clarify the associations between asymmetry during straight-line gait and the time difference in circular gait among subjects with greater gait asymmetry, such as for subjects afflicted with hemiparesis and unilateral musculoskeletal injury, and the association between the gait asymmetry and fall risk. In conclusion, the results of the present study demonstrated that the circular gait 
asymmetry was significantly higher in older subjects than that in younger subjects. In

addition, there was a significant relationship between the difference in the step length

during straight-line gait and gait time difference during circular gait in older adults.

These findings suggest that the circular gait test has the potential to better detect the gait laterality of older adults.

\section{Disclosure statement}

The authors declare no conflict of interest. 


\section{References}

[1] Hill K, Schwarz J, Flicker L, Carroll S. Falls among healthy, community-dwelling, older women: a prospective study of frequency, circumstances, consequences and prediction accuracy. Aust NZ J Public Health 1999; 23: 41-8.

[2] Yogev G, Plotnik M, Peretz C, Giladi N, Hausdorff JM. Gait asymmetry in patients with Parkinson's disease and elderly fallers: when does the bilateral coordination of gait require attention? Exp Brain Res 2007; 177: 336-46.

[3] Bautmans I, Jansen B, Van Keymolen B, Mets T. Reliability and clinical correlates of 3D-accelerometry based gait analysis outcomes according to age and fall-risk. Gait

Posture 2011; 33: 366-72.

[4] Chen IH, Yang YR, Chan RC, Wang RY. Turning-based treadmill training improves turning performance and gait symmetry after stroke. Neurorehabil Neural Repair 2014; 28: 45-55.

[5] Courtine G, Schieppati M. Human walking along a curved path. II. Gait features and EMG patterns. Eur J Neurosci 2003; 18: 191-205.

[6] Courtine G, Papaxanthis C, Schieppati M. Coordinated modulation of locomotor muscle synergies constructs straight-ahead and curvilinear walking in humans. Exp Brain Res 2006; 170: 320-35.

[7] Orendurff MS, Segal AD, Berge JS, Flick KC, Spanier D, Klute GK. The kinematics and kinetics of turning: limb asymmetries associated with walking a circular path. Gait Posture 2006; 23: 106-11.

[8] Guideline for the prevention of falls in older persons. American Geriatrics Society, British Geriatrics Society, and American Academy of Orthopaedic Surgeons Panel on Falls Prevention. JAm Geriatr Soc 2001; 49: 664-72.

[9] Shimada H, Suzukawa M, Tiedemann A, Kobayashi K, Yoshida H, Suzuki T. Which neuromuscular or cognitive test is the optimal screening tool to predict falls in frail community-dwelling older people? Gerontology 2009; 55: 532-8.

[10] Abellan van Kan G, Rolland Y, Andrieu S, et al. Gait speed at usual pace as a predictor of adverse outcomes in community-dwelling older people an International Academy on Nutrition and Aging (IANA) Task Force. J Nutr Health Aging 2009; 13: 881-9. [11] Maki BE. Gait changes in older adults: predictors of falls or indicators of fear. $J$ Am Geriatr Soc 1997; 45: 313-20.

[12] Hess RJ, Brach JS, Piva SR, VanSwearingen JM. Walking skill can be assessed in older adults: validity of the Figure-of-8 Walk Test. Phys Ther 2010; 90: 89-99.

[13] Sadeghi H, Allard P, Prince F, Labelle H. Symmetry and limb dominance in able- 
bodied gait: a review. Gait Posture 2000; 12: 34-45.

[14] Laroche DP, Cook SB, Mackala K. Strength asymmetry increases gait asymmetry and variability in older women. Med Sci Sports Exerc 2012; 44: 2172-81.

[15] Talbot LA, Musiol RJ, Witham EK, Metter EJ. Falls in young, middle-aged and older community dwelling adults: Perceived cause, environmental factors and injury. BMC Public Health 2005; 5 .

[16] Melzer I, Benjuya N, Kaplanski J. Postural stability in the elderly: a comparison between fallers and non-fallers. Age Ageing 2004; 33: 602-607. 
Table 1 Comparison of the young and older adults

\begin{tabular}{lcc}
\hline & $\begin{array}{c}\text { Young adults } \\
(\mathrm{n}=20)\end{array}$ & $\begin{array}{c}\text { Older adults } \\
(\mathrm{n}=25)\end{array}$ \\
\hline Straight-line gait & & \\
$\quad$ Speed (m/s) & $1.32 \pm 0.27$ & $0.97 \pm 0.24^{* *}$ \\
$\quad$ Longer step length between left and right (m) & $0.70 \pm 0.12$ & $0.55 \pm 0.12^{* *}$ \\
$\quad$ Shorter step length between left and right (m) & $0.65 \pm 0.13$ & $0.49 \pm 0.12^{* *}$ \\
Asymmetry index in step length (\%) & $8.35 \pm 7.13$ & $10.2 \pm 7.53$ \\
$\quad$ Longer stance duration between left and right (s) & $0.53 \pm 0.04$ & $0.56 \pm 0.11$ \\
$\quad$ Shorter stance duration between left and right (s) & $0.50 \pm 0.03$ & $0.54 \pm 0.10$ \\
Asymmetry index in stance duration (\%) & $4.08 \pm 5.84$ & $4.04 \pm 3.48$ \\
& & \\
Circular gait & & \\
$\quad$ Faster time between left and right directions (s) & $8.72 \pm 1.08$ & $15.4 \pm 6.81^{* *}$ \\
Slower time between left and right directions (s) & $9.04 \pm 1.13$ & $17.0 \pm 7.60^{* *}$ \\
Asymmetry index in circular gait time (\%) & $3.58 \pm 2.24$ & $9.06 \pm 7.05^{* *}$ \\
& & \\
\hline
\end{tabular}


Table 2 Correlation coefficient of gait asymmetry in a straight-line and circular gait

\begin{tabular}{|c|c|c|c|c|}
\hline \multirow[b]{2}{*}{ Circular gait } & \multicolumn{2}{|c|}{$\begin{array}{l}\text { Young adults } \\
\qquad \underline{(\mathrm{n}=20)}\end{array}$} & \multicolumn{2}{|l|}{$\begin{array}{l}\text { Older adults } \\
\qquad \underline{(\mathrm{n}=25)}\end{array}$} \\
\hline & $\frac{\text { Side-to-side }}{\text { difference }}$ & $\begin{array}{c}\text { Asymmetry } \\
\text { index }\end{array}$ & $\frac{\text { Side-to-side }}{\text { difference }}$ & $\begin{array}{l}\text { Asymmetry } \\
\text { index }\end{array}$ \\
\hline \multicolumn{5}{|l|}{ Straight-line gait } \\
\hline step length & 0.205 & 0.194 & $0.404^{*}$ & 0.168 \\
\hline stance duration & 0.046 & 0.172 & -0.297 & -0.039 \\
\hline
\end{tabular}

$*: p<0.05$ 